\author{
Agnieszka Kulczyńska \\ University of Warsaw \\ ul. Dobra 55 \\ 00-312 Warsaw, Poland \\ a.kulczynska@uw.edu.pl \\ http://orcid.org/0000-0001-8273-7185
}

\title{
L'hypallage ou le déplacement d'attribution dans le roman de Jerzy Andrzejewski Wielki Tydzień et dans sa traduction française
}

\begin{abstract}
The paper examines some linguistic aspects of Jerzy Andrzejewski's novel Wielki Tydzień, and compares them with the French version of the text. It focuses on the figure of hypallage, used recurrently by Andrzejewski. Usually defined as a transferred epithet, hypallage can also be conceived of as a partly fixed expression whose elements have regained - in a specific context - their combinatorial freedom. The paper describes the different functions of this type of transfer in the Polish original. It also examines the attitude adopted by the French translator regarding these unusual co-occurrences.

Keywords: hypallage, transferred attribute, modifier, unconventional cooccurrence
\end{abstract}

Nous nous proposons d'examiner certains aspects langagiers du roman de Jerzy Andrzejewski Wielki Tydzień publié en polonais en 1946, et de les confronter avec la version française du roman, due à Wladimir Vinaver, intitulée Sous le regard des autres et publiée en $1986 \mathrm{chez}$ 
Balland. Nous nous intéresserons notamment à la figure d'hypallage, employée de façon recurrente par Andrzejewski. Définie habituellement comme un déplacement d'attribution, l'hypallage peut être également conçue comme une séquence figée, ou du moins attendue, à laquelle on a rendu - dans un contexte d'énonciation spécifique - sa liberté combinatoire. Nous chercherons à décrire les différentes fonctions que ce type de transfert syntaxico-sémantique peut avoir dans l'original littéraire polonais. Nous nous pencherons également sur l'attitude adoptée par le traducteur français face à ces appariements inattendus.

1. Définition de l'hypallage

Employer une hypallage consiste à transférer une caractérisation (Gaudin-Bordes \& Salvan, 2008, p. 15), à déplacer « la qualification convenant à un nom sur un nom voisin » (Bonhomme, 1998, p. 31), à «rapporter à certains mots d'un énoncé ce qui devrait logiquement être attribué à d'autres », comme dans innocente lumière de ses yeux ou encore dans orgueil muet (Bergez, Géraud, \& Robrieux 2008, p. 112). Ce qui la distingue de la métaphore et de la métonymie, c'est que les deux termes associés y sont employés au sens propre. L'éventuelle surprise et le caractère inédit de la représentation induite résultent d'un choix syntaxique: il s'agit donc d'une figure de construction, et non d'un trope (encore que cette question ait été nuancée par Collins, 2012). Comme le résume Desurmont (2007, p. 2) :

L'hypallage se caractérise (...) par la conjonction de deux traits : la juxtaposition syntaxique de constituants dont l'un est le support de l'incidence de l'autre (typiquement, un nom et un adjectif), et la non-conformité de l'objet né de cette association.

\section{Variétés d'hypallage}

Une première approche classificatoire prend en compte l'aspect morphologique et s'intéresse aux types de rapports syntaxiques dans lesquels l'hypallage peut être instanciée. Selon cette optique - jugée quelquefois peu ambitieuse (Rastier, 2001, p. 120) - le décalage de 
qualification affecte majoritairement les adjectifs épithètes, mais peut également affecter les compléments de nom (Bonhomme, 1998, p. 31). Selon de nombreux auteurs, cependant, l'hypallage peut être instanciée dans tout rapport syntaxique (Ziomek, 1990, p. 225; Rastier, 2001, p. 120, Desurmont, 2007, p. 2).

Une autre tentative de classement pourra s'intéresser au degré d'originalité de l'hypallage et distinguera l'hypallage lexicalisée, utilisée dans la langue courante et qui «passe inaperçue » (comme sleepless night ou happy day, Desurmont, 2007, p. 2). On la discernera de l'hypallage vive, ressentie comme une figure, comme une représentation inédite, quelquefois analysable également en termes de métaphore ou de métonymie (Ziomek, 1990, p. 225).

Une troisième approche s'intéressera enfin à l'étendue du contexte qu'il faut prendre en compte pour interpréter une hypallage. GaudinBordes et Salvan (2008, p. 15) rappellent que le propre du mécanisme hypallagique réside dans le fait qu'un caractérisant (C), qui est incident sur le plan syntaxique à un élément $(\mathrm{E})$, mais qui forme avec lui une caractérisation non pertinente, se rattache aussi sémantiquement à un élément $\left(E^{1}\right)$, qui lui est contigu et qui, lui, pourrait être caractérisé par (C) de façon recevable.

Cependant, la contiguïté peut être entendue de deux manières. Au sens strict d'abord, elle signifie que l'élément caractérisé $\left(E^{1}\right)$ est présent dans le contexte immédiat du caractérisant (C), qu'il pourrait le régir syntaxiquement et que par conséquent, on a «simplement» affaire à un déplacement ou à une permutation syntaxiques. Mais la contiguïté peut aussi être entendue au sens large : dans ce cas, pour interpréter l'hypallage, il faut déceler la présence de l'élément caractérisé $\left(\mathrm{E}^{1}\right)$ dans un contexte plus vaste, celui d'un paragraphe ou même du texte dans son ensemble. Dans ce cas, cet élément n'est pas présent, mais impliqué par le contexte, donc restituable, voire purement interprétatif (Gaudin-Bordes \& Salvan, 2008, pp. 15-16).

\section{Parcours interprétatifs}

Comment décode-t-on une hypallage ? Une fois de plus, la figure révèle son caractère paradoxal. Autant il est tout à fait légitime de dire 
qu'elle n'entraîne en principe pas de doute sur le sens, malgré l'allotopie entre le caractérisé et le caractérisant (Bergez et al., 2008, p. 112; Desurmont, 2013, p. 19), autant il est tout aussi fondé d'affirmer qu'elle pose des problèmes délicats, et ceci dès l'étape de l'identification de la figure, sans parler de son interprétation ou de sa traduction (Ziomek, 1990, p. 224; Rastier. 2001, p. 121; Desurmont, 2007; Potok, 2008, p. 176).

Qu'elle soit problématique ou pas, l'interprétation de l'hypallage passe, dans une certaine mesure, par la restitution de la structure rationnelle, celle où la syntaxe et la logique concordent. Cette restitution nécessite que l'on identifie, dans le co-texte ou le contexte plus ou moins immédiat, l'élément qualifié $\left(\mathrm{E}^{1}\right)$ auquel se rattache sémantiquement l'élément qualifiant (C), pourtant rattaché syntaxiquement à l'élément (E).

Certes, la démarche interprétative qui consiste à «remettre les choses à leur place » (Rastier, 2001, p. 123) est réductrice, car toute glose, toute rationalisation détruit l'image (Desurmont, 2007, p. 3). On peut donc postuler une démarche interprétative qui ne soit ni réductrice (s'arrêtant au sens rationnel), ni productrice (ne retenant que le sens paradoxal), mais critique, prenant en compte les deux niveaux, c'est-à-dire sauvegardant l'anomalie de la représentation, tout en identifiant les mécanismes de transferts opérés (Rastier 2001: 124).

De quels mécanismes de transfert, de quels parcours interprétatifs s'agit-il? J. Ziomek (1990, p. 224) rappelle que la rhétorique classique distinguait trois types d'hypallage : celle des rapports temporels, celle des rapports propriété - propriétaire et celle des rapports cause conséquence. Desurmont (2006, pp. 167-174; 2013, p. 105 et s.) tente d'élargir l'inventaire des parcours conceptuels qui président à l'encodage et au décodage de la figure hypallagique, et énumère, outre les trois mécanismes mentionnés, les hypallages qui jouent sur la confusion des différents plans dans le champ visuel, sur la confusion entre l'objet référentiel et l'objet mental, sur la confusion entre la perception temporelle et spatiale, sur la confusion entre le sujet et l'objet et enfin sur la confusion des événements successifs. 
Ajoutons qu'à propos de chacun de ces mécanismes conceptuels, il est légitime de s'interroger sur l'éventuel transfert métonymique ou métaphorique, car celui-ci accompagne bien souvent l'hypallage.

4. Effets de sens de l'hypallage

A quoi sert l'hypallage lorsqu'elle constitue une figure vive et non pas un appariement lexicalisé et, par conséquent, imperceptible pour les utilisateurs de la langue?

Tout d'abord, on remarque le caractère nouveau, surprenant, de l'objet induit par l'hypallage, et le fait qu'elle en offre une représentation inédite, quelquefois poétique (Sienkiewicz, 2002, p. 215; Desurmont, 2007, pp. 2-3).

Dans un second temps, il convient de souligner le caractère subjectif de l'hypallage, traduisant la singularité d'une vision, ce qui conduit certains auteurs à qualifier cette figure d'impressionniste (Fromilhague \& Sancier-Château, 1996, p. 162; Bonhomme, 1998, p. 31). En effet, l'hypallage met au premier plan un regard, une conscience, et relègue au second plan l'objet référentiel dans son objectivité (Desurmont, 2006, p. 174; Desurmont, 2007, p. 1). A ce propos, Potok (2008, p. 175) rappelle une dénomination pittoresque de l'hypallage en espagnol: contagio imaginativo («contagion imaginaire »), et Sienkiewicz (2002, p. 214) insiste sur le caractère constitutif d'une conscience qui trouve toujours son expression dans cette figure.

Un troisième effet de sens porté potentiellement par l'hypallage est celui de synthèse, d'osmose, d'intrication de différents plans (comme dans « le silence blanc de l'hiver », cité par Bonhomme, 1998, p. 31). On pourra parler à ce propos de synesthésie, de décloisonnement des catégories du réel, d'annulation de frontières conceptuelles, d'appréhension psychologique de l'univers sur un mode d'inclusion (Bonhomme, 1998, p. 31; Desurmont, 2006, p. 174; Desurmont, 2007, pp. 4, 7; Potok, 2008, p. 180). C'est précisément parce qu'elle modifie le mode de pensée que certains auteurs (dont Sienkiewicz, 2002, p. 215) voient dans l'hypallage une figure de construction, certes, mais qui se double d'une figure de pensée. 
L'esthétique du flou, le caractère brouillé des frontières, sont autant d'effets pouvant surgir de l'emploi de l'hypallage (Bonhomme, 1998, pp. 31-32).

On relèvera enfin, et surtout, le caractère a-normal, non conforme de l'hypallage, qui trouble l'ordre, qui heurte le bon sens, en offrant une image déraisonnable et en s'écartant des représentations consensuelles. Si l'hypallage laisse entrevoir la doxa, le stéréotype, c'est pour les dépasser, pour s'attaquer à eux, voire pour les détruire. Elle présente par conséquent un caractère éminemment paradoxal (Rastier, 2001, pp. 123-125; Desurmont, 2007, pp. 2-3; GaudinBordes \& Salvan, 2008, p. 18).

Pour toutes ces raisons, l'hypallage qui - sur le plan microsyntaxique - est sans aucun doute irrecevable et non pertinente (Desurmont, 2007, p. 3), acquiert un caractère «hyperpertinent » sur le plan discursif et référentiel (Gaudin-Bordes \& Salvan, 2008, pp. 17, 19).

5. Nature et fonctions des hypallages choisies dans le roman Wielki Tydzień et leur traitement dans la traduction française

Considérons à présent quelques exemples d'hypallages relevées dans le roman de Jerzy Andrzejewski Wielki Tydzień et dans sa traduction française, Sous le regard des autres, en rappelant d'abord le contexte dans lequel elles apparaissent.

Les événements du roman se déroulent en 1943, pendant la période de Pâques, au moment où éclate l'insurrection au ghetto de Varsovie. Une jeune femme d'origine juive, Irena, trouve refuge chez une famille polonaise, les Malecki, ses amis d'avant la guerre. Jan Malecki et sa femme habitent un quartier résidentiel de Varsovie, éloigné du centre de la ville, et par conséquent du ghetto. Ils cherchent à cacher la présence d'Irena aux voisins de leur immeuble, ils cherchent surtout à cacher l'identité juive de leur hôte - mais en vain. Chassée par ses voisins, Irena dirigera ses pas vers le ghetto, dans un geste suicidaire.

L'action se situe en partie dans les quartiers centraux de Varsovie, dans les rues voisines du ghetto, où l'insurrection est brutalement écrasée par les Allemands. Les événements insurrectionnels sont 
relatés d'un point de vue extérieur, celui des passants polonais qui ne peuvent que déduire, à partir de différents indices, ce qui se passe derrière les murs du ghetto.

L'action se déroule, d'autre part, dans le quartier périphérique qu'habitent les Malecki et où Irena a trouvé momentanément refuge. Le soulèvement du ghetto et l'écrasement progressif de celui-ci sont là encore relatés d'un point de vue de témoins éloignés, qui perçoivent la tragédie en cours et mesurent son immensité à travers, par exemple, les lueurs des incendies qui éclairent la nuit, les fumées qui couvrent le ciel pendant la journée ou encore à travers les détonations qui font trembler la terre sous leurs pieds.

Cette restriction du champ de vision - ou plutôt de perception, car la vue n'est ni le seul, ni le principal sens sollicité - est une caractéristique essentielle de la narration d'Andrzejewski. Elle aboutit à l'émergence de la litote, cette figure qui «feint d'atténuer l'expression d'une réalité pour lui donner plus d'énergie » (Bonhomme, 1998: 78). Ainsi, le narrateur peut ne pas représenter l'horreur - ou du moins ne pas la représenter systématiquement - mais la suggérer, notamment à travers des procédés métonymiques dont nous venons de donner des exemples.

Autant sur le plan macrostructurel, ou narratif, la figure dominante est la métonymie au service de la litote, autant sur le plan microstructurel, c'est l'hypallage qui frappe par sa présence récurrente. Nous chercherons à cerner la nature et les fonctions de quelques-unes de ces hypallages, ainsi que le traitement qu'elles ont subi dans la traduction française du roman. Nous choisirons des passages où la figure sert à évoquer les événements du ghetto ou à peindre les états psychologiques des personnages qui y sont confrontés.

Voici le premier exemple (les initiales J.A. désignent l'original polonais ; les initiales W.V., la traduction publiée en français) :

(...) Pod murami getta było pusto. A ponad nimi, milczące i ciężkie, wznosiły się owe wysokie ściany kamienic. $\mathrm{Z}$ wąskimi okienkami i łamaną linią dachów wcinającą się w chmurne niebo, przypominały ogromną fortecę. 
Ludzie, ośmieleni spokojem, poczęli przystawać i przyglądać się samotnym ścianom. Naraz posypały się stamtąd wystrzały. (...) (J.A., p. 20)

(...) De l'autre côté de la place, silencieux et lourds, s'élevaient les hauts murs des immeubles juifs. Avec leurs meurtières, leur ligne crénelée, leur toits rejoignant le ciel couvert, ils ressemblaient à une énorme forteresse.

Ceux que l'accalmie rassurait s'arrêtèrent pour observer les murs. Soudain les coups de feu claquèrent à nouveau. (...) (W.V., p. 21)

Dans l'original polonais, nous avons affaire à une hypallage instanciée dans le rapport entre un nom et un adjectif épithète. Dans l'appariement inattendu samotnym ścianom (littéralement: murs solitaires), les deux termes sont à prendre au sens propre; pour remédier à l'anomalie qu'ils constituent, pour résoudre leur « énigme », il faut chercher dans le contexte un troisième élément, qui soit sémantiquement rattachable au caractérisant samotnym. Cet élément est absent du contexte immédiat, il devrait donc être restituable: en effet, on peut supposer que l'élément manquant $\left(\mathrm{E}^{1}\right)$ auquel se rattache sémantiquement l'adjectif solitaires sont les gens dont on devine la présence derrière les murs observés par les passants.

Le parcours interprétatif est donc de type métonymique. Si on cherche à le cerner avec plus de précision, c'est-à-dire à déterminer la nature de la «confusion » dont parle Ch. Desurmont (2006: 168), on observe que le transfert opère entre l'objet référentiel et l'objet mental, c'est-à-dire entre les murs que l'observateur voit et les gens dont il devine la présence.

Il s'agit bien d'une hypallage formant une image originale, qui a pour fonction de traduire une vision subjective, de rendre compte de la présence d'un regard et d'une conscience - situés, il est vrai, dans un foyer perceptif indéterminé, celui du narrateur ou celui des passants. Mais elle sert également à brouiller les catégories, à effacer la frontière entre le non humain et l'humain : sont-ce les murs qui ont acquis le trait d'humanité ou bien sont-ce les êtres humains qui en ont été privés ? Telle est la question portée par cette hypallage, question à laquelle il n'y a pas de réponse, l'hypallage étant, comme l'a montré Rastier (2001), indécidable. 
Dans la version française du roman, le traducteur a choisi d'omettre le qualificatif décalé samotnym: il a préféré rationaliser la représentation en supprimant l'hypallage. Vu la complexité des sens véhiculés par celle-ci, on peut regretter ce choix, d'autant qu'il n'y a pas de contraintes linguistiques qui pèseraient en sa faveur.

Considérons un autre passage :

Pachniało wiosną. $\mathrm{Z}$ bardzo daleka dochodziły miarowo pulsujące odgłosy wystrzałów. Noc dygotała nimi bez przerwy.

- Słyszysz?

- Tak, szepnęła.

Im dłużej nasłuchiwał, tym groźniej i surowiej obnażało się przed nim niespokojne drżenie ptonacej nocy (J.A., p. 97).

Cela sentait le printemps. De très loin, parvenait le bruit des combats. La nuit tout entière crépitait. Plus il écoutait, plus menaçant et sévère se dévoilait, devant lui, le tremblement de la nuit de feu (W.V., p. 93).

Le passage original comporte plusieurs hypallages, considérons d'abord celle qui est instanciée entre un nom et un complément de nom : drżenie [...] nocy (fr. tremblement de la nuit [...]). Il y a bien hypallage car, à proprement parler, la nuit ne peut pas trembler : le prédicat $(\mathrm{C})$, qui la caractérise, est donc non pertinent et il est légitime de rechercher, dans le contexte, un autre élément, $\left(\mathrm{E}^{1}\right)$, auquel il se rapporterait de façon plus rationnelle. Cet élément est aisément restituable : il se trouve dans le co-texte relativement proche, où sont évoqués les bruits de combats qui parviennent de très loin aux observateurs, et où on parle de la nuit tout entière qui crépite. La glose rationnelle (inévitablement réductrice) conduit donc à identifier $\left(\mathrm{E}^{1}\right)$ comme l'air dans lequel se propage le bruit lointain et continu des détonations, produisant un effet de tremblement. Le parcours interprétatif est là encore métonymique (nuit pour air de nuit); la confusion concerne les différents plans dans le champ perceptif. Cette hypallage constitue une figure vive.

Quelles sont ses fonctions? Tout d'abord, elle offre une vision globale, synthétique - voire synesthésique - des choses : un type de bruit et un type de mouvement se confondent, on perçoit à la fois le 
phénomène observé (le bruit des combats) et le milieu dans lequel il se propage, appréhendés habituellement par deux sens différents.

Mais cette lecture hypallagique n'est pas la seule possible, elle peut s'accompagner d'une lecture métaphorique. En effet, le constituant nominal opère à la manière d'une animisation : la nuit, qui tremble, se voit dotée d'un corps semblable à celui d'un être vivant. Le sens de cette métaphore se précise davantage grâce à la présence, dans le passage analysé, de l'isotopie de la peur. L'original polonais évoque „,niespokojne drżenie [...] nocy » (notons que l'adjectif niespokojne, qui pourrait être rendu en français par «inquiet», «tourmenté », «agité », «angoissé » ou "anxieux », a disparu de la traduction française). Le réseau sémantique de la peur est tissé aussi - de façon métonymique - par la tournure «Noc dygotała nimi bez przerwy », littéralement: "La nuit en grelottait / en frissonnait sans discontinuer ». La traduction française renonce à induire cette isotopie facultative et propose à sa place la formule (hypallagique): «La nuit tout entière crépitait $»$.

La nuit est ainsi, dans le texte polonais, assimilée indirectement à un être vivant, qui peut être parcouru de frissons et éprouver des émotions telles que l'inquiétude ou l'angoisse. Cette image, qui résulte d'une lecture métaphorique se superposant à la lecture hypallagique, est beaucoup moins nette dans la traduction française.

Examinons une autre hypallage présente dans le même fragment du texte, dans le constituant nominal «płonacej nocy» (littéralement : «la nuit en feu»). A proprement parler, il n'est pas possible que la nuit brûle, il y a donc hypallage. Si on tente de rationaliser l'image en cherchant un élément $\left(E^{1}\right)$ contigu et sémantiquement plus pertinent, on le trouve dans le contexte de l'ensemble de l'oeuvre : c'est bien le ghetto qui brûle, de nuit comme de jour. L'hypallage procède par un amalgame proche de la métonymie, en confondant l'événement et le moment de celui-ci. Il y a aussi une confusion entre l'objet référentiel et l'objet mental : les observateurs ne perçoivent que la lueur des incendies, mais savent que c'est le ghetto qui brûle.

Parler de la «nuit en feu» au lieu du «ghetto en feu» permet plusieurs effets de sens. Tout d'abord, on rend ainsi compte d'une 
vision particulière, localisée dans un foyer perceptif précis, celui d'un observateur nocturne et éloigné dans l'espace. D'autre part, la formule hypallagique permet d'appréhender le phénomène du ghetto insurgé non pas de façon analytique et distanciée, mais englobante et inclusive : la nuit «concerne » tout le monde, personne n'y échappe, on ne peut pas prendre ses distances avec elle... Enfin, le déplacement d'attribution propre à l'hypallage permet de faire jouer des connotations négatives du lexème «nuit», telles que les ténèbres, prises aussi bien au sens propre qu'au sens figuré.

Remarquons que dans la version française, le caractérisant polonais «płonacej nocy » a été rendu par le syntagme prépositionnel « nuit de $f e u »$. Pour sauvegarder l'hypallage, il aurait fallu que les deux termes du groupe soient interprétables dans leur sens ordinaire - or ce n'est pas le cas de «nuit de feu », qui désigne, métaphoriquement, une révélation religieuse ou intellectuelle.

Résumons: l'image globale qui émerge de l'ensemble de la formule polonaise «niespokojne drżenie ptonacej nocy» (littéralement : «tremblement inquiet de la nuit en feu ») est celle d'un phénomène à la fois sinistre, grandiose et impliquant tous ceux qui en sont témoins. Phénomène profondément troublant, menaçant, anormal, que l'on ne peut ni ignorer ni circonscrire dans l'espace, et par lequel on est irrémédiablement concerné.

Voici un autre passage, situé au tout début du récit: Malecki apprend la situation dramatique d'Irena, l'ayant rencontrée par hasard dans une des rues voisines du ghetto.

Zrobiło mu się przykro, jak jeszcze nigdy w ciągu całej znajomości z Ireną. Poczuł się straszliwie zawstydzony i upokorzony jej losem, a także swoją bezradnością i swoim uprzywilejowaniem (J.A., p. 27).

Il se sentit gêné comme jamais depuis qu'il connaissait Irène, humilié de la voir ainsi, honteux de son impuissance et de sa situation privilégiée (W.V., p. 27)

Le passage original comporte une caractérisation non pertinente, ou du moins problématique: upokorzony jej losem (littéralement: humilié par son sort). Cette fois, l'hypallage est instanciée entre un verbe au passif et un complément d'agent. Autant le groupe voisin : honteux de 
son impuissance et de sa situation privilégiée reste conforme à la vision communément partagée, autant humilié par son sort pose problème et déclenche le mécanisme évoqué ci-dessus, celui de la recherche d'un élément qui pourrait être caractérisé de façon plus pertinente par l'élément en question. Selon la glose rationnelle, l'humiliation concerne surtout Irena: c'est elle qui a le droit de se sentir humiliée par le sort qui est le sien.

$\mathrm{Ne}$ pas en parler directement, déplacer l'attribution vers Malecki, est un acte générateur de sens. L'hypallage acquiert ici une fonction de litote : les mots sont en deçà de ce qu'ils signifient en réalité. On représente l'humiliation d'Irena de façon détournée; par la même formule, on évoque la compassion de Malecki sans la nommer explicitement - mais ce sont des «moins » qui « réveillent l'idée du plus $\gg$ (Jaubert 2008).

L'hypallage est par ailleurs, comme nous l'avons dit, une figure anti-doxale ; elle interroge, dépasse ou altère la vision communément partagée. Compatir à l'humiliation de quelqu'un est une image consensuelle. Etre humilié par l'humiliation d'autrui dépasse cette vision ordinaire et permet de peindre un état psychologique dans toute sa complexité.

La traduction française a préservé cette image : l'hypallage a été sauvegardée, même si syntaxiquement, le complément d'agent (humilié par son sort) est devenu un complément circonstanciel de cause (humilié de la voir ainsi).

\section{Conclusion}

Cet aperçu, quoique très sélectif, illustre la nature morphologiquement variée de l'hypallage: les exemples analysés comportent des hypallages instanciées entre un nom et un adjectif épithète, mais aussi entre un nom et un complément de nom, ou encore entre un verbe au passif et un complément d'agent. Ces aspects morphologiques restent pourtant secondaires: comme le dit Rastier (2001, p. 120), «le problème que pose l'hypallage ne concerne pas les classes morphologiques, mais le franchissement des frontières entre syntagmes ». 
Les exemples illustrent également différents «stades» de la réalisation de la figure d'hypallage décrits par Gaudin-Bordes \& Salvan (2008) : le terme caractérisé $\left(E^{1}\right)$ y est tantôt effectivement présent dans le contexte proche, tantôt restituable à partir d'un contexte moins immédiat. Ces distinctions pourraient être également analysées en termes d'hypallage in praesentia et in absentia.

On a également pu observer une variété de parcours interprétatifs. Il semble pourtant peu réaliste de chercher à dresser une liste exhaustive des mécanismes qui président à l'encodage et au décodage de la figure hypallagique. Tout comme la métonymie - caractérisée par Rastier (2001, p. 125) comme une «classe de problèmes hétérogènes plutôt qu'une figure » - l'hypallage semble reposer sur un nombre potentiellement illimité de types de transferts.

Les exemples analysés démontrent la diversité des fonctions remplies par l'hypallage. En dehors de l'extraordinaire économie qui lui est propre et qui permet d'appréhender des données de l'univers dans un raccourci synthétique, voire poétique, l'hypallage sert aussi, dans le matériel analysé, à présenter les événements sur un mode inclusif, de sorte à rendre indirectement compte des émotions éprouvées par les témoins de l'anéantissement du ghetto. Elle permet également de représenter la complexité des situations et des réactions psychologiques qu'elles suscitent.

Mais la fonction de l'hypallage à laquelle nous aimerions nous arrêter est celle de la litote. Celle-ci s'avère en effet présente chez Andrzejewski non seulement sur le plan macrostructurel, ou narratif, mais aussi sur le plan microstructurel, et son émergence est possible grâce à l'hypallage. Ainsi, l'appariement murs solitaires opère d'une façon paradoxale: en apparence, il passe sous silence les gens emprisonnés dans le ghetto ; en réalité, il met leur présence en relief, car c'est cette donnée, qui apparaît au terme d'un travail raisonné de mise en relation, qui sauve la cohérence du passage. Lorsqu'il parle de la nuit en feu, le narrateur choisit de ne pas évoquer le ghetto explicitement; pourtant, ce choix ne conduit pas à évacuer ni à minimiser l'image du drame, mais au contraire, à élargir celle-ci bien au-delà des limites imposées par la vision communément partagée. 
Dans l'hypallage humilié par son sort, on évite de nommer le sentiment de compassion, mais paradoxalement, on rend compte d'une compassion portée à son plus haut degré.

Les figures, rappelons-le, ne sont pas des ornements, mais des « formes d'expression qui contribuent à la mise en oeuvre du projet porté par le texte » (Fromilhague \& Sancier, 1996, p. 186). Il semble que le projet porté par le roman d'Andrzejewski soit de montrer un monde disloqué et appréhendé par un langage qui est disloqué luimême; de rendre compte de l'horreur en employant des mots qui sont en deçà de ce qu'ils disent en réalité; de représenter le caractère extrêmement complexe des émotions éprouvées par des témoins de la tragédie. L'hypallage sert parfaitement ces projets.

En ce qui concerne les stratégies de traduction, les exemples analysés montrent une fois de plus que tout renoncement à l'hypallage de la part du traducteur - qu'il s'agisse d'omission ou de rationalisation - appauvrit le sens original. Ramener le texte « dans le droit chemin » de la doxa, c'est-à-dire de l'attendu, de l'habituel, c'est annuler les différents sens véhiculés par l'hypallage, avec leur nouveauté et leur complexité. Quelquefois les contraintes de la langue d'arrivée constituent un obstacle insurmontable, mais lorsque ce n'est pas le cas, il est souhaitable de préserver la figure.

Pour ce faire, il faut d'abord la reconnaître et l'interpréter. La reconnaissance de l'hypallage passe par la conscience que les deux termes qui forment une caractérisation non pertinente sont à prendre au sens propre, même si une lecture métaphorique parallèle n'est pas exclue. L'interprétation, elle, suppose la recherche d'un élément syntaxiquement indépendant, mais sémantiquement rattaché au caractérisant. L'identification de cet élément dans le contexte est parfois une condition nécessaire pour choisir de façon adéquate parmi les équivalents potentiels de caractérisant (C) dans la langue d'arrivée.

Bref, l'hypallage exige que l'on « [lise] ce qui est écrit » (Rastier, 2001, p. 118). On ne saurait mieux dire. 
Bibliographie

Bergez, D., Géraud V., \& Robrieux J. J. (2008). Vocabulaire de l'analyse littéraire. Paris: Dunod.

Bonhomme, M. (1998). Les figures clés du discours. Paris: Seuil.

Collins, H. (2012). La relative transparence de l'hypallage : l'hypallage est-elle un trope ? Etudes de Stylistique Anglaise, 5, 43-60.

Desurmont, Ch. (2006). Les figures de l'hypallage. Bulletin de la Société de Stylistique Anglaise, 27, 159-176.

Desurmont, Ch. (2007). Adjectif composé et figures. Palimpsestes, 19, 119-134. DOI: 10.4000/palimpsestes. 126 .

Desurmont, Ch. (2013). Les ressources de linéarité: l'exemple de l'hypallage. Bulletin de la Société de Stylistique Anglaise, 6, 105-113.

Fromilhague, C., \& Sancier-Chateau A. (1996). Introduction à l'analyse stylistique. Paris: Dunod.

Gaudin-Bordes, L., \& Salvan, G. (2008). Le sens en marche : le cas de l'hypallage. L'information grammaticale, 116, 15-19. DOI: 10.3406/igram.2008.3942.

Jaubert, A. (2008). Dire et plus ou moins dire. Analyse pragmatique de l'euphémisme et de la litote. Langue française, 160(4), 105-116. DOI: 10.3917/lf.160.0105.

Potok, M. (2008). Hypallage jako problem poetyki przekładu na przykładzie romanc Federico Garcii Lorki. Przektadaniec, 17, 173-185.

Rastier, F. (2001). Indécidable hypallage. Langue Française, 129, 111-127. DOI: 10.3406/lfr.2001.1021.

Sienkiewicz, B. (2002). Prawdziwy koniec hypallage?. Teksty drugie, 3, 212-222.

Ziomek, J. (1990). Retoryka opisowa. Wrocław, Warszawa, Kraków: Zakład Narodowy im. Ossolińskich Wydawnictwo. 